\title{
A Class of Locally Complete Intersection Multiple Structures on Smooth Algebraic Varieties as Support
}

\author{
Nicolae Manolache
}

June 7, 2018

Math. Sub. Class.: Primary 14M05, 13H10

\begin{abstract}
The systematic study of multiple structures, made necessary (and possible) by the notion of scheme introduced by Grothendieck, began with the papers $[\mathrm{Fo}, \mathrm{Fe}$ and was continuated in BF1] and $\mathrm{M} 1, \mathrm{M} 2, \mathrm{M}$ ] (the last three use also ideeas from $\mathrm{PS}]$ ). In [BF1, BF2], along the classification, up to multiplicity 4 , of multiple locally complete intersection (lci for short) structures on a smooth curve embedded in a smooth threefold, general classes of multiple structures are introduced, the so-called "primitive" and "quasiprimitive" structures. The primitive ones are characterized by the fact that, locally, they are defined by equations of the type $x^{n}=0, y=0, z=0, \ldots, u=0$; the quasiprimitive ones are those which are generically primitive. The general study of these structures was continued by several authors, from which we mention [Bo], Dr1], Dr2], [Dr3], Dr4].

In this paper we give the construction of a class of multiple locally complete intersection (lci for short) structures on a smooth algebraic variety as support. This class contains the lci structures defined locally by the "the next" monomial equations, namely those which are defined locally by equations of the form $x^{n}=0, y^{2}=0, z=0, \ldots, u=0$.
\end{abstract}

\section{Preliminaries}

Let $X$ be a smooth connected algebraic variety over an algebraically closed field $k$. A (locally) Cohen-Macaulay scheme $Y$ is called a multiple structure on $X$ if the subjacent reduced scheme $Y_{\text {red }}$ is $X$. In this case all the local rings of $Y$ have the same multiplicity (cf. [M2]), which is called the multiplicity of $Y$. Let $Y$ be embedded in a smooth variety $P$. Let $I$ be the (sheaf) ideal of $X$ in $P$ and $J$ be the ideal of $Y$ in $P$. Let $m$ be the positive integer such that $I^{m} \not \subset J$, $I^{m+1} \subset J$. To $Y$ one associates canonically three filtrations. They are:

1. Let $I^{(\ell)}$ be the ideal obtained throwing away the embedded components of 
$I^{\ell}+J$ and let $Z_{\ell}$ be the corresponding scheme. This gives the Bănic $\breve{a}$-Forster filtration (cf. [BF2):

$$
\begin{array}{ccccccccccc}
\mathcal{O}_{Y}=I^{(0)} & \supset & I=I^{(1)} & \supset & I^{(2)} & \supset & \ldots & \supset & I^{(m)} & \supset & I^{(m+1)}=0 \\
& X=Z_{1} & \subset & Z_{2} & \subset & \ldots & \subset & Z_{m} & \subset & Z_{m+1}=Y
\end{array}
$$

$Z_{\ell}$ are not, in general, Cohen-Macaulay. But this is true if $\operatorname{dim}(X)=1$. The graded associated object $\mathcal{B}(Y)=\bigoplus_{\ell=0}^{m} I^{(\ell)} / I^{(\ell+1)}$ is naturally a graded $\mathcal{O}_{X^{-}}$ algebra. If the schemes $Z_{\ell}$ are Cohen-Macaulay, the graded components of $\mathcal{B}(Y)$ are locally free sheaves on $X$.

2. Let $X_{\ell}$ be defined by $I_{\ell}=J: I^{m+1-\ell}$. When $X_{\ell}$ are Cohen-Macaulay, the quotients $I_{\ell} / I_{\ell+1}$ are locally free sheaves on $X$. Again, if $\operatorname{dim}(X)=1, X_{\ell}$ are Cohen-Macaulay. This is also true if $Y$ is lci (i.e. locally complete intersection) of multiplicity at most 6 (cf. [M2]). In general this is not always the case. This filtration was considered in M1].

3. Let $Y_{\ell}$ be the scheme given by $J_{\ell}=J: I_{m+1-\ell}=J:\left(J: I^{\ell}\right)$. When $X_{\ell}$ is Cohen-Macaulay, $Y_{\ell}$ has the same property. The graded object $\mathcal{A}(Y)=$ $\bigoplus_{\ell=0}^{m} J_{\ell} / J_{\ell+1}$ is a graded $\mathcal{O}_{X}$-algebra and $\mathcal{M}(Y)=\bigoplus_{\ell=0}^{m} I_{\ell} / I_{\ell+1}$ is a graded $\mathcal{A}(Y)$-module. This filtration was considered in [M2].

The system of the graded components $\left(\mathcal{A}_{0}(Y), \ldots \mathcal{A}_{m}(Y) ; \mathcal{M}_{0}(Y), \ldots \mathcal{M}_{m}(Y)\right)$ is called the type of $Y$. $Y$ is called of free type when all the graded pieces are locally free. As already remarked, in dimension 1 , or if $Y$ is lci of multiplicity up to 6 , this is the case.

The previous filtrations have the following properties:

1) In general the above filtrations are different. Take for instance $X=\operatorname{Spec}(k)$, $Y=\operatorname{Spec}\left(k[x, y] /\left(x^{3}, x y, y^{4}\right)\right), P=\operatorname{Spec}(k[x, y])$

2) $Z_{\ell} \subset Y_{\ell} \subset X_{\ell}$

2') there are canonical morphisms: $\mathcal{B}(Y) \rightarrow \mathcal{A}(Y) \rightarrow \mathcal{M}(Y)$

3) The multiplications

$$
\begin{aligned}
& \mathcal{A}_{\ell_{1}}(Y) \otimes \mathcal{A}_{\ell_{2}}(Y) \quad \rightarrow \quad \mathcal{A}_{\ell_{1}+\ell_{2}}(Y) \\
& \mathcal{A}_{\ell_{1}}(Y) \otimes \mathcal{M}_{\ell_{2}}(Y) \rightarrow \mathcal{M}_{\ell_{1}+\ell_{2}}(Y)
\end{aligned}
$$

are never the zero maps for $\ell_{1}, \ell_{2} \geq 0, \ell_{1}+\ell_{2} \leq m$ (cf. [M2]).

4) From the definitions, one has the exact sequences:

$$
\begin{aligned}
0 & \rightarrow \mathcal{M}_{\ell}(Y) \rightarrow \mathcal{O}_{X_{\ell+1}} \rightarrow \mathcal{O}_{X_{\ell}} \rightarrow 0 \\
0 & \rightarrow \mathcal{A}_{\ell}(Y) \rightarrow \mathcal{O}_{Y_{\ell+1}} \rightarrow \mathcal{O}_{Y_{\ell}} \rightarrow 0
\end{aligned}
$$

5) If $Y$ is Gorenstein of free type, then $X_{\ell}$ and $Y_{m+1-\ell}$ are locally algebraically linked (cf. [M1). In particular one has the exact sequences:

$$
\begin{gathered}
0 \rightarrow \omega_{X_{m+1-\ell}} \otimes \omega_{Y}^{-1} \rightarrow \mathcal{O}_{Y} \rightarrow \mathcal{O}_{Y_{\ell}} \rightarrow 0 \\
0 \rightarrow \omega_{Y_{m+1-\ell}} \otimes \omega_{Y}^{-1} \rightarrow \mathcal{O}_{Y} \rightarrow \mathcal{O}_{X_{\ell}} \rightarrow 0
\end{gathered}
$$

6) Let $Y$ be a free type Cohen-Macaulay multiple structure on a smooth support $X$. 
Then (cf M3] $Y$ is Gorenstein if and only if the following conditions are fulfilled

(a) $\mathcal{A}_{m}(Y)$ and $\mathcal{M}_{m}(Y)$ are line bundles

(b) $\mathcal{A}_{m}(Y)=\mathcal{M}_{m}(Y)$

(c) The canonical maps:

$$
\mathcal{A}_{\ell}(Y) \rightarrow \mathcal{H o m}_{\mathcal{O}_{X}}\left(\mathcal{M}_{m-\ell}(Y), \mathcal{M}_{m}(Y)\right) \cong \mathcal{M}_{m-\ell}(Y)^{\vee} \otimes \mathcal{M}_{m}(Y)
$$

are isomorphisms.

6') In particular: if $Y$ is Gorenstein of free type, then (cf also [M2]):

$$
\begin{aligned}
& \text { (a) } \operatorname{rank} \mathcal{A}_{\ell}(\mathrm{Y})=\operatorname{rank} \mathcal{M}_{\mathrm{m}-\ell}(\mathrm{Y}) \\
& \text { (b) } \mathcal{A}_{\ell}(Y)=\mathcal{M}_{\ell}(Y) \text { iff } \operatorname{rank} \mathcal{A}_{\ell}(\mathrm{Y})=\operatorname{rank} \mathcal{A}_{\mathrm{m}-\ell}(\mathrm{Y})
\end{aligned}
$$

In this paper all the schemes are algebraic schemes over a fixed algebraically closed field $k$, of characteristic 0 .

\section{Some structures of multiplicity $2 \mathrm{n}$}

Definitions 1. 1. Let $E$ be a rank 2 vector bundle on $X$ and $L$ be a line bundle on $X$. If $s$ is a section of $L \otimes S^{2} E$, locally of the form $\ell \otimes\left(a e_{1}^{2}+2 b e_{1} e_{2}+c e_{2}^{2}\right)$, ( $\ell$ a local generator of $L$ and $e_{1}, e_{2}$ local generators for $\left.E\right)$, then $\delta(s) \in \Gamma\left(X, L^{2} \otimes(\operatorname{det} E)^{2}\right)$ is the section defined locally by $\left(b^{2}-a c\right) \ell^{2} \otimes\left(e_{1} \wedge e_{2}\right)^{2}$.

When $s \in \mathcal{H o m}\left(L, S^{2} E\right)$ define $\delta(s)=\delta\left(s \otimes 1_{L^{-1}}\right) \in \Gamma\left(X, L^{-2} \otimes(\operatorname{det} E)^{2}\right)$.

When $s \in \mathcal{H o m}\left(S^{2} E, L\right)$, define $\delta(s)=\delta\left(s^{\vee}\right) \in \Gamma\left(X, L^{2} \otimes(\operatorname{det} E)^{-2}\right)$.

Extend this definition also to a surjection $\varphi: S^{2} E \rightarrow F$, where $F$ is a vector bundle of rank 2. Namely, if $s_{\varphi}: L \rightarrow S^{2} E$ is the kernel of $\varphi$, then put $\delta(\varphi)=\delta\left(s_{\varphi}\right)$.

2. With $L$ and $E$ as above, for $s \in \Gamma\left(X, L \otimes S^{n} E\right)$, define the Hessian $h(s) \in \Gamma\left(X, L^{2} \otimes(\operatorname{det} E)^{2} \otimes S^{2 n-4} E\right)$ in the obvious way: if $s$ is given locally by $\ell \otimes \sum\left(\begin{array}{c}n \\ i\end{array}\right) a_{i j} e_{1}^{i} e_{2}^{j}$, then $h(s)$ is locally the Hessian of this symmetric form.

When $s \in \operatorname{Hom}\left(L, S^{n} E\right)$ define $h(s)=h\left(s \otimes 1_{L^{-1}}\right) \in \Gamma\left(X, L^{-2} \otimes(\operatorname{det} E)^{2} \otimes\right.$ $\left.S^{2 n-4} E\right)$

When $s \in H \operatorname{Hom}\left(S^{n} E, L\right)$ define $h(s)=h\left(s^{\vee}\right) \in \Gamma\left(X, L^{2} \otimes(\operatorname{det} E)^{-2} \otimes S^{2 n-4} E^{\vee}\right)$

Theorem 2.1. Let $X \subset P$ be smooth varieties and let $I$ be the ideal sheaf of $X$ in $P$. The following construction produces a lci multiple structure $Y$ with support $X$, of multiplicity $2 n$.

Step 1. Take a rank 2 vector bundle $E_{1}$ on $X$, a surjection $p_{1}: I / I^{2} \rightarrow E_{1}$ and define $I_{2}:=\operatorname{ker}\left(I \rightarrow I / I^{2} \rightarrow E_{1}\right)$. Then it follows:

$$
S^{2} E_{1} \cong I^{2} / I I_{2}, \quad S^{3} E_{1} \cong I^{3} / I^{2} I_{2} \quad \text { etc. }
$$

Step 2. Take $E_{2}$ a rank 2 vector bundle on $X$ and $p_{2}: I_{2} / I I_{2} \rightarrow E_{2}$, such that the map $\mu_{2}: S^{2} E_{1}=I^{2} / I I_{2} \hookrightarrow I_{2} / I I_{2} \rightarrow E_{2}$ is surjective. Take $I_{3}=\operatorname{ker}\left(I_{2} \rightarrow\right.$ $\left.I_{2} / I I_{2} \rightarrow E_{2}\right)$. Then $E_{3}:=I I_{2} / I I_{3}$ is a rank 2 vector bundle on $X$. 
Step 3. Take a surjection $p_{3}: I_{3} / I I_{3} \rightarrow E_{3}$ such that the composition of $p_{3}$ with the natural inclusion $i_{3}: I_{2} / I I_{3} \rightarrow I_{3} / I I_{3}$ is an isomorphism of vector bundles. Define $I_{4}=\operatorname{ker}\left(I_{3} \rightarrow I_{3} / I I_{3} \rightarrow E_{3}\right)$. Then $E_{4}:=I I_{3} / I I_{4}$ is a rank 2 vector bundle on $X$.

Step $k . \quad(4 \leq k \leq n-2)$ Take a surjection $p_{k}: I_{k} / I I_{k} \rightarrow E_{k}$ such that the composition of $p_{k}$ with the natural inclusion $i_{k}: E_{k}=I I_{k-1} / I I_{k} \rightarrow I_{k} / I I_{k}$ is an isomorphism of vector bundles. Define $I_{k+1}=\operatorname{ker}\left(I_{k} \rightarrow I_{k} / I I_{k} \rightarrow E_{k}\right)$. Then $E_{k+1}:=I I_{k} / I I_{k+1}$ is a rank 2 vector bundle on $X$.

Step $n$-1. Take a surjection $p_{n-1}: I_{n-1} / I I_{n-1} \rightarrow E_{n-1}$ such that the composition of $p_{n-1}$ with the natural inclusion $i_{n-1}: E_{n-1}=I I_{n-2} / I I_{n-1} \rightarrow$ $I_{n-1} / I I_{n-1}$ is an isomorphism of vector bundles. Define $I_{n}=\operatorname{ker}\left(I_{n-1} \rightarrow\right.$ $\left.I_{n-1} / I I_{n-1} \rightarrow E_{n-1}\right)$.

Step $n$. Take a line bundle $L$ on $X$ such that $c_{2 n-3}\left(L^{2} \otimes\left(\operatorname{det} E_{1}\right)^{-2} \otimes S^{2 n-4} E_{1}^{\vee}\right)=$ 0 . Take a surjection $p_{n}: I_{n} / I I_{n} \rightarrow L$ such that $\mu_{n}:=p_{n} j_{n}$ is surjective, where $j_{n}$ is the canonical map $j_{n}: S^{n} E_{1} \rightarrow I_{n} / I I_{n}$ and such that the Hessian of $\mu_{n}$ vanishes nowhere. Define $I_{n+1}:=\operatorname{ker}\left(I_{n} \rightarrow I / I I_{n} \rightarrow L\right)$. Then $I_{n+1}$ defines a subscheme $Y$ of $P$ which is lci of multiplicity $2 n$.

One has: $\omega_{Y} \cong \omega_{X} \otimes L^{-1}$ and $\mathcal{B}(Y)=\mathcal{A}(Y)=\mathcal{M}(Y)=\mathcal{O}_{X} \oplus E_{1} \oplus \ldots \oplus$ $E_{n-1} \oplus L$. Moreover, by [M3], the canonical maps $E_{\ell} \rightarrow \mathcal{H o m}\left(E_{n-\ell}, L\right)$ are isomorphisms.

Proof. We will determine at each step the local equations of the scheme defined by $I_{\ell}$, i.e. the equations in the completions of the local rings with respect to their maximal ideals.

Step 1. It is clear that in each point of $X$ there are local parameters $x, y, z, \ldots u$ which define the scheme $X$ in that point such that the ideal $I_{2}$ is of the form $\left(x^{2}, x y, y^{2}, z, \ldots u\right)$. As $E_{1}=I / I_{2}$, the multiplication map

$$
E_{1} \otimes E_{1} \cong \frac{I}{I_{2}} \otimes \frac{I}{I_{2}} \rightarrow \frac{I^{2}}{I I_{2}}
$$

factors through $E_{1} \otimes E_{1} \rightarrow S^{2} E_{1}$ and thus the map $S^{2} E_{1} \rightarrow I^{2} / I I_{2}$, as a surjection between rank 3 vector bundles is an isomorphism. In the same way one shows all the other isomorphisms.

Step 2. A local computation shows that the following three situations can occur:

a) $\delta\left(\mu_{2}\right)=0$. Then, in convenient local coordinates, $I_{3}=\left(x^{3}, x^{2} y, y^{2}, z, \ldots, u\right)$. a') $\delta\left(\mu_{2}\right)$ defines an effective nonzero divisor $D$. Then in the points of this divisor, in convenient coordinates, $I_{3}=\left(x^{3}, x^{2} y, y^{2}+\alpha_{2} x^{2}, z, \ldots, u\right)$, where $\alpha_{2}=0$ is the local equation of $D$. 
b) $\delta\left(\mu_{2}\right)$ vanishes nowhere. After a change of variables, one has $I_{3}=\left(x^{3}, x y, y^{3}, z, \ldots u\right)$.

In all these situations, by local computations, one shows that $E_{3}:=I I_{2} / I I_{3}$ is a rank 2 vector bundle.

Step 3. We treat in the same time the situations a) and a') from step 2. One shows easily that, in new local coordinates (i.e. new generators of the maximal ideal): $I_{4}=\left(x^{4}, x^{3} y, y^{2}+\alpha_{2} x^{2}+\alpha_{3} x^{3}, z, \ldots, u\right)$ In the case $\left.\mathrm{b}\right)$, one obtains $I_{4}=\left(x^{4}, y^{4}, x y-\lambda x^{3}-\mu y^{3}, z, \ldots, u\right)$. Via the change of coordinates $x=X+\mu Y^{2}, y=Y+\lambda X^{2}$, one gets $I_{4}=\left(X^{4}, Y^{4}, X Y, z, \ldots, u\right)$. In both cases, by local computations, one shows that $E_{4}:=I I_{3} / I I_{4}$ is a rank 2 vector bundle.

Step $k .(4 \leq k \leq n-2)$ By induction, at steps $k-1$ and $k-2$ we get two possibilities for the local shape of our ideals:

a) $I_{k}=\left(x^{k}, x^{k-1} y, y^{2}+\alpha_{2} x^{2}+\ldots+\alpha_{k-1} x^{k-1}, z, \ldots, u\right)$,

$$
I_{k-1}=\left(x^{k-1}, x^{k-2} y, y^{2}+\alpha_{2} x^{2}+\ldots+\alpha_{k-2} x^{k-2}, z, \ldots, u\right)
$$

or

b) $I_{k}=\left(x^{k}, x y, y^{k}, z, \ldots, u\right)$, $I_{k-1}=\left(x^{k-1}, x y, y^{k-1}, z, \ldots, u\right)$,

In case a), as the local generators of $E_{k}=I I_{k-1} / I I_{k}$ are $x^{k}, x^{k-1} y$, one obtains, in convenient coordinates, $I_{k+1}=\left(x^{k+1}, x^{k} y, y^{2}+\alpha_{2} x^{2}+\ldots+\alpha_{k} x^{k}, z, \ldots, u\right)$

In case b), as the local generators of $E_{k}=I I_{k-1} / I I_{k}$ are $x^{k}, y^{k}$ one obtains $I_{k+1}=\left(x^{k+1}, x y-\lambda x^{k}-\mu y^{k}, y^{k+1}, x^{2} y, x y^{2}, z, \ldots, u\right)$. With the change $y=Y+$ $\lambda x^{k-1}$ one gets $I_{k+1}=\left(x^{k+1}, x Y-\mu\left(Y+\lambda x^{k-1}\right)^{k},\left(Y+\lambda x^{k-1}\right)^{k+1}, x^{2} Y, x Y^{2}, z, \ldots, u\right)$, where $z, \ldots u$ are changed tacitly. Now, taking $x=X+\mu Y^{k-1}$, one gets $I_{k+1}=\left(X^{k+1}, X Y, Y^{k+1}, z, \ldots, u\right)$

In both cases, by local computations, one shows that $E_{k+1}:=I I_{k-1} / I I_{k}$ is a rank 2 vector bundle on $X$.

Step $n-1$ is similar with the previous one, but we are no more interested in the vector bundle $E_{n}:=I I_{n-1} / I I_{n}$.

Step $n$. We have two cases:

a) $I_{n}=\left(x^{n}, x^{n-1} y, y^{2}+\alpha_{2} x^{2}+\ldots+\alpha_{n-1} x^{n-1}, z, \ldots, u\right)$,

or

b) $I_{n}=\left(x^{n}, x y, y^{n}, z, \ldots, u\right)$.

Take $\ell$ to be the local generator of the line bundle $L$.

In case a), $\mu_{n}$ will have the shape:

$$
\mu_{n}\left(x^{n-2 p} y^{2 p}\right)=\left(-\alpha_{2}\right)^{p} r \ell, \quad \mu_{n}\left(x^{n-2 p-1} y^{2 p+1}\right)=\left(-\alpha_{2}\right)^{p} s \ell
$$

A direct computation of the Hessian of $\mu_{n}$ gives:

$$
h\left(\mu_{n}\right) \equiv-(n-1)^{2} s^{2} \ell^{2} x^{2 n-4} \bmod \alpha_{2}
$$

From here it follows that the condition upon Hessian implies that $s$ is invertible and then, after some change of coordinates:

$$
I_{n+1}=\left(x^{n}, y^{2}+\alpha_{2} x^{2}+\ldots+\alpha_{n-1} x^{n-1}, z, \ldots, u\right)
$$


b) In this case the application $\mu_{n}$ has the form:

$$
\mu_{n}\left(x^{n}\right)=r \ell, \quad \mu_{n}\left(y^{n}\right)=s \ell, \quad \mu_{n}\left(x^{n-p} y^{p}\right)=0 \text { for } p \neq 0 \text { or } p \neq n
$$

Then the Hessian will be:

$$
h\left(\mu_{n}\right)=n^{2}(n-1)^{2} r s \ell^{2} x^{n-2} y^{n-2},
$$

which shows that both $r$ and $s$ should be invertible.

Then, after a change of coordinates:

$$
I_{n+1}=\left(x^{n}+y^{n}, x y, z, \ldots, u\right)
$$

Remark 2.2. Conversely, all multiple structures $Y$ on a smooth variety $X$ embedded in a smooth variety $P$, given locally by ideals of the form $J=$ $\left(x^{n}, y^{2}+\alpha_{2} x^{2}+\ldots+\alpha_{n-1} x^{n-1}, z, \ldots, u\right)$, or $J=\left(x^{n}+y^{n}, x y, z, \ldots, u\right)$ are obtainable by the procedure described in the above theorem.

Remark 2.3. The case $n=2$ for curves in threefolds is treated in [BF1, [BF2], the case $n=3$ is covered in $\mathrm{M} 2$.

Example 2.4. The above construction has almost no degree of liberty in codimension 2. We shall exemplify this for the case of a line embedded in $\mathbb{P}^{3}$. Take $X$ to be a line defined by the homogeneous ideal $I=(x, y)$ and consider $u, v$ to be the homogeneous coordinates on $X$. We shall describe the above construction for $n=2$ (whence multiplicity 4 ) and $n=3$ (which gives a multiplicity 6 structure).

Case $n=2 . E_{1}$ has to be $I / I^{2}=2 \mathcal{O}_{X}(-1)$ and then $I_{2}=\left(x^{2}, x y, y^{2}\right)$. The condition on $L$ is fullfilled only by $L=\mathcal{O}_{X}(-1)$. Then $I_{3}=\left(x^{2}, y^{2}\right)$, in new coordinates.

Case $n=3 . E_{1}$ and $I_{2}$ are as above. The condition on the Hessian of $\mu_{3}$ determines the line bundle $L: L=\mathcal{O}_{X}(-3)$. Then $E_{2} \cong E_{1}^{\vee} \otimes L=2 \mathcal{O}_{X}(-2)$. The ideal $I_{3}$ will have the shape $I_{3}=\left(c_{1} x^{2}+c_{2} x y+c_{3} y^{2}, x^{3}, x^{2} u, x y^{2}, y^{3}\right)$, where $c_{1}, c_{2}, c_{3}$ are constants. After a change of the homogeneous coordinates in $\mathbb{P}^{3}$ this ideal takes one of the shapes: $I_{3}=\left(x^{2}, x y^{2}, y^{3}\right)$ or $I_{3}=\left(x^{3}, x y, y^{3}\right)$. Accordingly, for $I_{4}$ there are two possibilities: $I_{4}=\left(x^{2}, y^{3}\right)$ or $I_{4}=\left(x y, x^{3}+y^{3}\right)$.

In the case of codimension greater than 2 one can give examples which are not globally complete intersections. The simplest are with $n=2$.

Example 2.5. Consider a line $X$ embedded in $\mathbb{P}^{4}$. Then:

$$
\frac{I}{I^{2}}=\frac{(x, y, z)}{(x, y, z)^{2}}=3 \mathcal{O}_{X}(-1)
$$

We take $E_{1}=\mathcal{O}_{X}(-1) \oplus \mathcal{O}_{X}(r)$, with $r \geq 0$. Consider $p_{1}: I / I^{2} \rightarrow E_{1}$ given by a matrix of the shape

$$
\left(\begin{array}{ccc}
0 & 0 & 1 \\
-b & a & 0
\end{array}\right),
$$


where $a, b$ are forms of degree $r+1$ in the homogeneous coordinates $u, v$ on $X$, without common zeroes on $X$. Then $I_{2}=\left(a x+b y, x^{2}, x y, x z, y z, y^{2}, z^{2}\right)$. The condition on the Hessian of $\mu_{2}$ determines $L=\mathcal{O}_{X}(r-1)$. We take $p_{2}$ such that the map $\mu_{2}: S^{2} E_{1}=\mathcal{O}_{X}(-2) \oplus \mathcal{O}_{X}(r-1) \oplus \mathcal{O}_{X}(2 r) \rightarrow L$ to be the projection on the second factor. Then $I_{3}=\left(a x+b y, x^{2}, x y, y^{2}, z^{2}\right)$

A similar example can be given for the case $\operatorname{dim} X=2$ :

Example 2.6. In $\mathbb{P}^{6}$ let $X$ be the plane given by the eqations $x=y=z=t=0$. If $u, v, w$ are homogeneous coordinates on $X$ and $a, b, c$ are forms in $u, v, w$ of the same degree $r+1$, then $Y$ defined by the ideal $I_{3}=(b x-a y, c y-b z, a z-$ $c x)+(x, y, z)^{2}+\left(t^{2}\right)$ is a multiple structure with $n=2$ on $X$.

Acknowledgment The author was partially supported by the CEEX Programme of the Romanian Ministry of Education and Research, contract 2CEx06-11-20/2006 and the Mittag-Leffler Institute. The author wants to express his warmest thanks for the hospitality and the wonderfull working atmosphere and conditions offered by the Mittag-Leffler Institute.

\section{References}

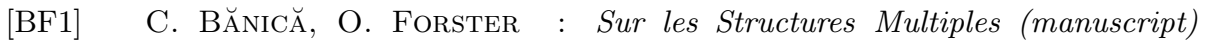
(1981)

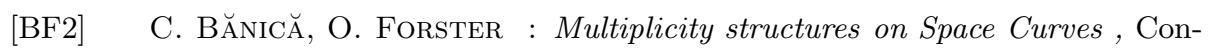
temporary mathematics , 58 (1986)

[Bo] M. Boratynsky : Locally Complete Intersection Multiple Structures on Smooth Algebraic Curves, Proc. of the Amer. math. Soc. , 115 (1992), p. $877-879$

[Dr1] JEAN-MARC DrÉzet : Faisceaux cohérents sur les courbes multiples , Collectanea Mathematica 57, 2 (2006) , p. 121-171

[Dr2] JEAN-MARC DrÉzet : Paramétrisation des courbes multiples primitives, Advances in Geometry 7 (2007) , p. 559-612.

[Dr3] JEAN-MARC DrÉZET : Moduli spaces of coherent sheaves on multiple curves in "Algebraic cycles, sheaves, shtukas, and moduli; Impanga lecture notes", Trends in Mathematics, Birkhuser (2008) , p. 33-44.

[Dr4] JEAN-MARC DRÉZET : Faisceaux sans torsion et faisceaux quasi localement libres sur les courbes multiples primitives, preprint (2008).

[Fe] D. Ferrand : Courbes Gauches et Fibrés de Rang 2, C.R. Acad. Sci. Paris , 281 (1975) , p. 345-347

[Fo] R. Fossum : Commutative Extensions by Canonical Modules are Gorenstein Rings , Proc. of the Amer. Math. soc. , 40 (1973) , p. 395-400

[M1] N. Manolache : Cohen-Macaulay Nilpotent Structures , Rev. Roumaine Math. pures et Appl. , 31 (1986) , p. 563-575 
[M2] N. Manolache : Multiple Structures on Smooth Support , Math. Nachr. , 167 (1994), p. 157-202

[M3] N. Manolache : Gorenstein Multiple Structures on Smooth Algebraic Varieties , arXiv:00706.2204v1 (mathAG).

[PS] C. Peskine, L. SzPiro : Liaison des Variétes Algébriques , Invent. Math., 26 (1977) , p. 271-302

Nicolae Manolache

Institute of Mathematics "Simion Stoilow"

of the Romanian Academy

P.O.Box 1-764 Bucharest, RO-014700

e-mail: nicolae.manolache@imar.ro 